\title{
Traumatismo dentário: avaliação da conduta dos Cirurgiões Dentistas que atuam em um pronto socorro odontológico municipal
}

Dental trauma: evaluation of the conduct of Dental surgeons who work in the municipal dental emergency room

Traumatismos dentales: evaluación de la conducta de los Cirujanos Dentistas que trabajan en las urgencias dentales municipales

\section{Resumo}

O objetivo deste estudo foi avaliar conduta e conhecimento dos profissionais cirurgiões-dentistas que atuam no pronto socorro odontológico municipal, sobre a proposta de tratamento dos agravos do ligamento periodontal depois de um trauma dentário. Trata-se de um trabalho descritivo com uma abordagem quantitativa. Foi-se realizado um estudo de natureza observacional transversal, com aplicação de questionário eletrônico, que foi composto por nove questões relacionadas ao perfil profissional e atuação diante do agravo do ligamento periodontal ocasionadas por um trauma dentário, exceto avulsão, validado por Pedrini em 2008 e adaptado para a plataforma do Google Forms. Pode-se verificar neste trabalho após análise dos dados, que dentre os pesquisados, a maioria era do sexo masculino, todos acima de 50 anos, e com aproximadamente 30 anos de formado e com formação predominante numa universidade privada. Quanto a especialização, 75,0\% dos profissionais são especialistas. Em relação à conduta do cirurgiãodentista se é adequada ou inadequada frente ao tratamento do traumatismo dentário, pode-se concluir que em concussão $(50,0 \%)$ foi inadequada e $(50,0 \%)$ adequada, na subluxação foi $100 \%$ inadequada, na luxação extrusiva $(50,0 \%)$ foi inadequada e $(50,0 \%)$ adequada, luxação lateral foi $100 \%$ adequada e luxação intrusiva $(75,0 \%)$ inadequada e $(25,0 \%)$ adequada. Portanto, verificou-se que os profissionais cirurgiões-dentistas não detêm conhecimento suficiente para atuar de modo apropriado aos danos causados no ligamento periodontal após um trauma dentário.

Palavras-chave: Cirurgiões-dentistas; Conhecimento; Conduta; Traumatismo dentário.

\begin{abstract}
The aim of this study was to evaluate the behavior and knowledge of dental surgeons who work in the municipal dental emergency room, regarding the proposal for treating periodontal ligament damage after dental trauma. This is a descriptive work with a quantitative approach. A cross-sectional observational study was carried out, with the application of an electronic questionnaire, which consisted of nine questions related to the professional profile and performance in the face of periodontal ligament damage caused by dental trauma, except for avulsion, validated by Pedrini in 2008 and adapted for the Google Forms platform. It can be seen in this work, after analyzing the data, that among those surveyed, most were male, all over 50 years old, with approximately 30 years of graduation and predominantly trained in a private university. As for specialization, 75.0\% of professionals are specialists. Regarding the dentist's conduct, whether it is adequate or inadequate in the treatment of dental trauma, it can be concluded that in concussion $(50.0 \%)$ it was inadequate and $(50.0 \%)$ adequate, in subluxation it was $100 \%$ inadequate, in the extrusive dislocation $(50.0 \%)$ it was inadequate and (50.0\%) adequate, lateral dislocation was $100 \%$ adequate and intrusive
\end{abstract}


dislocation $(75.0 \%)$ was inadequate and $(25.0 \%)$ adequate. Therefore, it was found that dental surgeons do not have enough knowledge to act appropriately to the damage caused to the periodontal ligament after dental trauma.

Keywords: Dentists; Dental trauma; Conduct; Knowledge.

\begin{abstract}
Resumen
El objetivo de este estudio fue evaluar el comportamiento y conocimiento de los cirujanos dentistas que laboran en el servicio de urgencias odontológicas municipales, sobre la propuesta de tratamiento del daño del ligamento periodontal posterior a un traumatismo dental. Se trata de un trabajo descriptivo con enfoque cuantitativo. Se realizó un estudio observacional transversal, con la aplicación de un cuestionario electrónico, el cual constaba de nueve preguntas relacionadas con el perfil y desempeño profesional ante el daño del ligamento periodontal por traumatismo dental, excepto avulsión, validado por Pedrini en 2008 y adaptado para la plataforma Google Forms. Se puede observar en este trabajo, luego de analizar los datos, que entre los encuestados, la mayoría eran hombres, todos mayores de 50 años, con aproximadamente 30 años de graduación y predominantemente formados en una universidad privada. En cuanto a la especialización, el 75,0\% de los profesionales son especialistas. En cuanto a la conducta del odontólogo, adecuada o inadecuada en el tratamiento del trauma dental, se puede concluir que en conmoción cerebral (50.0\%) fue inadecuada y $(50.0 \%)$ adecuada, en subluxación fue $100 \%$ inadecuada, en luxación extrusiva (50.0\%) fue inadecuada y (50.0\%) adecuada, la luxación lateral fue $100 \%$ adecuada y la luxación intrusiva (75.0\%) fue inadecuada y (25.0\%) adecuada. Por lo tanto, se encontró que los cirujanos dentales no tienen el conocimiento suficiente para actuar de manera adecuada ante el daño causado al ligamento periodontal después de un traumatismo dental.
\end{abstract}

Palabras clave: Cirujanos dentales; Conocimiento; Conducta; Traumatismo dental.

\title{
1. Introdução
}

Dentre os principais agravos da saúde pública mundial, pode-se citar o traumatismo dentário, o qual é tratado como uma injúria de proporção variável, podendo ser de origem acidental ou intencional. Ao contrário do que muitos acreditam, esse evento pode influenciar direta e indiretamente o bem-estar das pessoas (Souza et al., 2013).

Existem vários fatores associados à etiologia do trauma dentário, dentre eles pode-se citar as quedas, as atividades esportivas, os acidentes automobilísticos, a violência e, além desses, os aspectos anatômicos que favorecem a incidência de traumas dentários (Souza et al., 2013). Evidentemente a ocorrência de traumas é maior em jovens e ocorrem com maior frequência nos incisivos centrais superiores da dentição permanente, sendo justificado pelos fatores predisponentes (Conheca et al., 2006; Dantas et al., 2019).

Os traumatismos são divididos pelos que acometem tecidos duros dentais; trinca de esmalte; fratura de esmalte; fratura de esmalte e dentina; fratura de esmalte, dentina e polpa; fratura coronorradicular (com/sem exposição pulpar) e fratura radicular; e os que acometem estrutura de suporte, como concussão; subluxação; extrusão; luxação lateral; intrusão e avulsão (Conheca et al., 2006; Diangelis et al., 2012).

Desse modo, sabendo que os traumas na cavidade bucal podem variar de uma fratura de esmalte até a perda dentária, as condutas de atendimento também serão diferentes. O socorro normalmente é realizado pelo cirurgião dentista consultado no momento ou logo após a ocorrência do trauma (Sanabe et al., 2009). O plano de tratamento requer uma minuciosa análise, estabelecida por uma boa entrevista clínica, exame físico e radiográfico (Diangelis et al., 2012).

Certamente a previsão de sucesso desses traumatismos vai depender do atendimento emergencial oferecido pelo cirurgião-dentista, visto que a negligência ou imperícia pode acarretar inúmeras sequelas como: reabsorções internas e externas, sensibilidade, calcificações pulpares, necrose pulpar, anquilose dentária e até a perda dentária (Bastianini et al., 2021).

Equipes da família da atenção primária a saúde, principalmente, a equipe de saúde bucal encontra-se em situação favorável, pois possui acesso à população, seja no consultório, nas escolas ou comunidade e com isso tem possibilidade de treinamento para os demais profissionais e responsáveis em caso de emergência odontológica (Abu-Dawoud et al., 2007).

Diante disso, é de grande necessidade que os cirurgiões-dentistas possuam conhecimento atualizado sobre as condutas frente a um traumatismo dentário e assim pode-se contribuir para a melhoria do bem-estar da população. (Antunes et al., 2012). 
O objetivo deste estudo é avaliar a conduta e conhecimento dos profissionais cirurgiões-dentistas que atuam em um pronto socorro odontológico municipal, sobre o plano de tratamento dos agravos do ligamento periodontal após um trauma dentário.

\section{Metodologia}

Trata-se de um estudo observacional transversal. Segundo (Zangirolami-Raimundo, 2018), o objetivo do estudo transversal é obter dados seguros que ao final da pesquisa permitam elaborar resultantes confiáveis, além de gerar novas contingências que poderão ser investigadas com novas explorações. A obtenção dos dados foi realizada na Unidade de Pronto Atendimento - UPA de um município de Minas Gerais, com os profissionais cirurgiões-dentistas.

O estudo teve início após a pesquisa ser avaliada e aprovada pelo Comitê de Ética em Pesquisa do Centro Universitário de Patos de Minas - UNIPAM, sob protocolo 4.742.068 (CAAE 46551121.3.0000.5549).

Foi utilizado um questionário validado por Pedrini em 2008 e adaptado para a plataforma do Google Forms. O questionário eletrônico era composto por nove questões relacionadas ao perfil profissional e atuação diante do agravo do ligamento periodontal ocasionadas por um trauma dentário, exceto avulsão.

A pesquisa obteve-se uma taxa de resposta de $57,14 \%(n=4)$. Do total de 7 cirurgiões-dentistas plantonistas, 3 não quiseram participar da pesquisa. O serviço desses profissionais funciona durante todos os dias da semana e possui ao todo, sete habilitados, um para cada dia da semana, trabalhando em plantões de 16 horas. O estudo foi voltado para essa equipe. Para categorizar o grupo em estudo a estatística descritiva foi empregada, através de tabelas de frequências.

Após leitura e concordância do Termo de Consentimento Livre e Esclarecido teve início a pesquisa. Foram excluídos do estudo o profissional cirurgião-dentista que não faziam parte da unidade de pronto atendimento.

\section{Resultados}

Analisando os dados, observou-se que dentre os participantes, a maioria era do gênero masculino, todos acima de 50 anos, com aproximadamente 30 anos de formado e a formação predominante era universidade privada. Quanto à especialização, 75,0\% dos profissionais são especialistas.

Quando perguntado se atuavam em outras áreas além da sua especialidade, os cirurgiões-dentistas responderam que atuava em Clínica Geral (42,8\%), Cirurgia (14,3\%), Dentística (14,33\%), Prótese Dentária (14,33\%) e em Periodontia $(14,33 \%)$. Quando questionados sobre a capacidade de lidar com todos os tipos de injúrias do ligamento periodontal, causadas por traumatismo dentário, todos os profissionais afirmaram que sim (Tabela 1).

Tabela 1 - Frequência das classes dos parâmetros analisados no questionário aplicado a cirurgiões-dentistas que atuam no pronto socorro odontológico municipal.

\begin{tabular}{|c|c|c|}
\hline $\begin{array}{l}\text { Descrição dos parâmetros analisados no questionário } \\
\text { aplicado }\end{array}$ & Classes dos parâmetros analisados & Frequência \% (n) \\
\hline \multirow{2}{*}{ Ano de conclusão do seu curso de Graduação } & Antes 1990 & $75,0(3)$ \\
\hline & Depois 1990 & $25,0(1)$ \\
\hline \multirow{2}{*}{ Tipo de instuição } & Pública & $25,0(1)$ \\
\hline & Privada & $75,0(3)$ \\
\hline \multirow{2}{*}{ Idade } & Até 50 anos & $0,0(0)$ \\
\hline & Acima de 50 anos & $100,0(4)$ \\
\hline \multirow{2}{*}{ Gênero } & Masculino & $75,0(3)$ \\
\hline & Feminino & $25,0(1)$ \\
\hline
\end{tabular}


Research, Society and Development, v. 10, n. 16, e394101623819, 2021

(CC BY 4.0) | ISSN 2525-3409 | DOI: http://dx.doi.org/10.33448/rsd-v10i16.23819

\begin{tabular}{|c|c|c|}
\hline \multirow{2}{*}{ Possui especialização } & Sim & $75,0(3)$ \\
\hline & Não & $25,0(1)$ \\
\hline \multirow{2}{*}{ Além da sua especialidade, atua em outras áreas } & Sim & $100,0(3)$ \\
\hline & Não & $0,0(0)$ \\
\hline \multirow{5}{*}{ Atuação em outras áreas } & Clínica Geral & $42,8(3)$ \\
\hline & Cirurgia & $14,3(1)$ \\
\hline & Dentística & $14,3(1)$ \\
\hline & Periodontia & $14,3(1)$ \\
\hline & Prótese & $14,3(1)$ \\
\hline \multirow{4}{*}{ Plano(s) de tratamento para Concussão } & Acompanhamento & $50,0(4)$ \\
\hline & Exame radiográfico & $25,0(2)$ \\
\hline & Ajuste oclusal & $12,5(1)$ \\
\hline & Dieta líquida e pastosa por 15 dias & $12,5(1)$ \\
\hline $\begin{array}{l}\text { Descrição dos parâmetros analisados no questionário } \\
\text { aplicado }\end{array}$ & Classes dos parâmetros analisados & Frequência \% (n) \\
\hline \multirow{5}{*}{ Plano(s) de tratamento para Subluxação } & Acompanhamento & $40,0(4)$ \\
\hline & Exame radiográfico & $20,0(2)$ \\
\hline & Aguarda reerupção espontânea & $10,0(1)$ \\
\hline & Contenção dentária & $20,0(2)$ \\
\hline & Dieta líquida e pastosa por 15 dias & $10,0(1)$ \\
\hline \multirow{6}{*}{ Plano(s) de tratamento para Luxação extrusiva } & Reposicionamento dentário & $25,0(4)$ \\
\hline & Contenção dentária & $18,8(3)$ \\
\hline & Acompanhamento & $25,0(4)$ \\
\hline & Exame radiográfico & $18,8(3)$ \\
\hline & Tratamento endodôntico & $6,3(1)$ \\
\hline & Dieta líquida e pastosa & $6,3(1)$ \\
\hline \multirow{8}{*}{ Plano(s) de tratamento para Luxação lateral } & Exame radiográfico & $21,1(4)$ \\
\hline & Reposicionamento dentário & $15,8(3)$ \\
\hline & Contenção dentária, & $21,1(4)$ \\
\hline & Tratamento endodôntico & $5,3(1)$ \\
\hline & Acompanhamento & $21,1(4)$ \\
\hline & Antibioticoterapia & $5,3(1)$ \\
\hline & Dieta líquida e pastosa & $5,3(1)$ \\
\hline & Tratamento endodôntico & $5,3(1)$ \\
\hline \multirow{7}{*}{ Plano(s) de tratamento para Luxação intrusiva } & Exame radiográfico & $25,0(4)$ \\
\hline & Aguarda reerupção espontânea & $25,0(4)$ \\
\hline & Acompanhamento & $12,5(2)$ \\
\hline & Tratamento endodôntico & $12,5(2)$ \\
\hline & Tracionamento ortodôntico & $12,5(2)$ \\
\hline & Tracionamento cirúrgico & $6,3(1)$ \\
\hline & Reposicionamento dentário & $6,3(1)$ \\
\hline \multirow{2}{*}{$\begin{array}{l}\text { Você seria capaz de tratar todos os tipos de traumatismos } \\
\text { apresentados? }\end{array}$} & Sim & $100,0(4)$ \\
\hline & Não & $0,0(0)$ \\
\hline \multirow{2}{*}{$\begin{array}{l}\text { Você sugere outros tipos de tratamento para os } \\
\text { traumatismos acima? }\end{array}$} & $\operatorname{Sim}$ & $50,0(2)$ \\
\hline & Não & $50,0(2)$ \\
\hline
\end{tabular}

Fonte: Dados da pesquisa (2021). 
A Tabela 2 indica se a conduta do cirurgião dentista é adequada ou inadequada quanto ao tratamento dentoalveolar. Com relação à Concussão $(50,0 \%)$ inadequada e (50,0\%) adequada, na subluxação foi 100\% inadequada, na luxação extrusiva $(50,0 \%)$ foi inadequada e $(50,0 \%)$ adequada, luxação lateral foi $100 \%$ adequada e luxação intrusiva $(75,0 \%)$ foi inadequada e $(50,0 \%)$ adequada.

Tabela 2 - Avaliação da conduta adotada por cirurgiões dentistas que atuam no pronto socorro odontológico municipal diante de tratamento dos agravos do ligamento periodontal.

\begin{tabular}{lll}
\hline Classificação & $\begin{array}{l}\text { Tipos de Tratamento } \\
\text { Adequado - n (\%) }\end{array}$ & Inadequado - n (\%) \\
\hline Concussão & $2(50,0)$ & $2(50,0)$ \\
Subluxação & $0(0,0)$ & $4(100,0)$ \\
Luxação extrusive & $2(50,0)$ & $2(50,0)$ \\
Luxação lateral & $4(100,0)$ & $0(0,0)$ \\
Luxação intrusive & $1(25,0)$ & $3(75,0)$ \\
\hline
\end{tabular}

Fonte: Dados da pesquisa (2021).

A Tabela 3 mostra a classificação dos traumatismos, as características e as condutas correta, visto que adequado e inadequado foi julgado de acordo com a conduta como um todo, sem levar em consideração os procedimentos isolados.

Tabela 3 - Resposta sobre a conduta correta dos traumatismos que deveria ser adotada pelos cirurgiões dentistas.

\begin{tabular}{|c|c|c|}
\hline Classificação & Características & Condutas \\
\hline Concussão & $\begin{array}{l}\text { Lesão de tecidos de suporte sem perda ou } \\
\text { deslocamento do elemento dental. }\end{array}$ & $\begin{array}{l}\text { Exame radiográfico e o acompanhamento. Se o CD incluiu o ajuste oclusal e/ou } \\
\text { outros procedimentos pertinentes também foram considerados adequados. }\end{array}$ \\
\hline Subluxação & $\begin{array}{l}\text { Lesão de tecidos de suporte com presença de } \\
\text { hemorragia gengival. }\end{array}$ & $\begin{array}{l}\text { Exame radiográfico, o ajuste oclusal e o acompanhamento. Se o CD acrescentou a } \\
\text { contenção dentária por período de } 1 \text { a } 2 \text { semanas, a antibioticoterapia e/ou outros } \\
\text { procedimentos também foram considerados adequados. }\end{array}$ \\
\hline Luxação extrusiva & $\begin{array}{l}\text { O elemento dental se desloca parcialmente no } \\
\text { sentindo axial do alvéolo dental. Presença de } \\
\text { sangramento e aparência do dente alongado. }\end{array}$ & $\begin{array}{l}\text { Exame radiográfico, o reposicionamento dentário, a contenção dentária por } 2 \text { a } 3 \\
\text { semanas, o ajuste oclusal, a antibioticoterapia e o acompanhamento. Se o CD } \\
\text { incluiu o tratamento endodôntico e/ou outros procedimentos também foram } \\
\text { considerados corretos. }\end{array}$ \\
\hline Luxação lateral & $\begin{array}{l}\text { Deslocamento do elemento dental em relação } \\
\text { ao osso do processo alveolar. Clinicamente, a } \\
\text { coroa se apresenta encurtada e existe } \\
\text { sangramento gengival. }\end{array}$ & $\begin{array}{l}\text { Exame radiográfico, o reposicionamento dentário, a contenção dentária por período } \\
\text { de } 3 \text { a } 4 \text { semanas, o ajuste oclusal, a antibioticoterapia e o acompanhamento. Se o } \\
\text { CD incluiu o tratamento endodôntico e/ou outros procedimentos também foram } \\
\text { considerados corretos. }\end{array}$ \\
\hline Luxação intrusiva & $\begin{array}{l}\text { Perda total do elemento dental. Clinicamente, } \\
\text { o alvéolo dental fica vazio ou preenchido com } \\
\text { coágulo sanguíneo. }\end{array}$ & $\begin{array}{l}\text { Exame radiográfico, o tracionamento ortodôntico ou tracionamento cirúrgico, e } \\
\text { neste caso, a contenção dentária seria necessária por período de } 2 \text { a } 3 \text { semanas, o } \\
\text { ajuste oclusal, a antibioticoterapia, o tratamento endodôntico e o acompanhamento. } \\
\text { Como no questionário não foi citado se o dente possuía rizogênese incompleta ou } \\
\text { completa também foi considerada a alternativa de se aguardar a reerupção } \\
\text { espontânea. Se o CD incluiu o reposicionamento dentário e/ou outros } \\
\text { procedimentos também foram considerados corretos. }\end{array}$ \\
\hline
\end{tabular}

Fonte: Pedrini (2008).

\section{Discussão}

O traumatismo dentário pode ser definido como uma agressão térmica, química ou mecânica sofrida pelo dente e estruturas adjacentes, cuja magnitude supera a resistência encontrada nos tecidos ósseos e dentários e a sua ampliação tem relação direta com a intensidade, tipo e duração do impacto (Queiroz et al., 2019; Santos, 2020).

Os danos traumáticos são excepcionais e faz que o comportamento rápido e eficaz, frente ao trauma dentário, seja essencial. A fim de que o procedimento seja realizado de forma correta, é fundamental que seja feito um bom exame clínico e radiográfico instantaneamente (Pedrini, 2008; Losso, 2011; Carvalho et al., 2020). 
A experiência da abordagem, controle clínico e radiográfico nas ocorrências de trauma dental é de suma importância para manutenção e prognóstico favorável do elemento dentário (Pacheco, 2016). Os dados apontam que o uso da radiografia foi um dos tópicos mais indicado dentro da preparação do tratamento, independente da injúria. Esta conduta foi citada por todos os profissionais.

Na ocorrência de concussão e subluxação, o periodonto sofre apenas um trauma leve. Um dos principais sinais clínicos da subluxação é a existência de sangramento no sulco gengival (Flores, 2001). Um meio dos entrevistados, em concussão e todos os entrevistados em subluxação elaboraram estratégias de tratamento inadequadas para essas injúrias. Isso evidencia que quanto mais simples o traumatismo, mais difícil se tornou o protocolo de tratamento, maior as sequelas e pior o prognóstico.

A estrutura pulpar pode reagir de diversas formas mediante um dano traumático. A concussão e subluxação representam pequenas danificações à vascularização pulpar. A luxação extrusiva, a luxação lateral e a luxação intrusiva, apresentam um maior transtorno e com isso aumenta as lesões ao suprimento neurovascular pulpar o que dificulta a revascularização (Andreasen, 2001; Pedrini, 2008).

Os resultados para os padrões adotadas frente aos traumas listados mostrou que há uma incompreensão sobre a parte biológica fundamental já que as maiorias dos profissionais não souberam responder os padrões adequados. Corroborando com os estudos realizados por Pedrini (2008) e Santos (2018), onde parte dos especialistas entrevistados também não propuseram condutas adequadas a estes tipos de injúrias.

A luxação intrusiva e extrusiva obteve um reduzido número de planejamentos adequados. Alguns dos agravos causados pelas luxações (extrusiva e intrusiva) incluem necrose pulpar, reabsorção radicular inflamatória, anquilose, reabsorção por substituição e diminuição do suporte ósseo marginal (Andreasen, 2001).

Quando abordados eram aptos a atender diversos traumatismos, todos os entrevistados disseram que sim, diferente do estudo de Santos (2018), onde as maiorias dos cirurgiões-dentistas que colaboraram com a pesquisa responderam que não teriam capacidade para tratar todos os tipos de injurias do ligamento.

Outro aspecto avaliado nesse estudo foi o ajuste oclusal, visto que o reposicionamento nem sempre coloca o dente exatamente em seu local de origem e os contatos prematuros, podem ocasionar um trauma adicional (Panzarini, 2008; Mortele, 2016). Esse foi um item que frequentemente faltou na proposta de tratamento elaborada pelos profissionais e que quando não realizado pode contribuir negativamente para o prognóstico.

O acompanhamento de qualquer modelo de trauma é de extrema importância porque não é possível conhecer exatamente qual a extensão do dano provocado ao dente e seus tecidos de suporte e quais as consequências futuras (Andreasen, 2001). Dentre os planos de tratamento proposto, o acompanhamento foi um dos itens mais citados, independente do trauma.

Este estudo revelou que a experiência e conhecimento dos profissionais cirurgiões-dentistas entrevistados de um modo geral é baixo, apesar de existir na literatura protocolos que orientam sobre o tratamento mais adequado para os diversos tipos de traumatismo dentário. (Flores, 2001; Azevedo, 2012).

Mediante os dados obtidos com o estudo, fica perceptível a necessidade de aumentar estratégias para melhorar o domínio dos profissionais quanto ao algum traumatismo dentário com o intuito de melhorar a estimativa dessas lesões (Pedrini, 2008; Azevedo, 2012; Santos et al., 2018).

É de grande valor que os profissionais cirurgiões-dentistas possuam entendimento técnico, adequado e continuado sobre o tema em pauta, auxiliando então no bem-estar e qualidade assistência à população (Pedrini, 2008; Azevedo, 2012; Santos et al., 2018). 


\section{Conclusão}

Portanto, confirmou-se que os cirurgiões-dentistas não possuem entendimento suficiente para cuidar de modo apropriado das lesões mais severas do ligamento periodontal após traumatismo dentoalveolar.

Por sua vez, devem ser oferecidos aos cirurgiões-dentistas capacitações, com a intenção de manter estes profissionais atualizados em relação aos cuidados de injúrias de trauma alveolodentário.

Como sugestão para trabalhos futuro, uma vez observado que nem sempre as condutas foram adequadas, seria pesquisar as sequelas que foram desenvolvidas nesses dentes após o traumatismo.

\section{Referências}

Abu-Dawoud, M., Al-Enezi, B., \& Andersson, L. (2007). Knowledge of emergency management of avulsed teeth among young physicians and dentists. Dental traumatology: official publication of International Association for Dental Traumatology, 23(6), 348-355. https://doi.org/10.1111/j.16009657.2006.00477.x

Andreassen, J.O. (2001). Fundamentos de traumatismo dental: guia de tratamento passo a passo (2a ed). Artmed Editora.

Antunes, L. A. A., Leao, A. \& Maia, T. L. C. (2012). Impacto do traumatismo dentário na qualidade de vida de crianças e adolescentes: revisão crítica e instrumentos de medida. Ciênc. saúde coletiva, 17 (12). https://doi.org/10.1590/S1413-81232012001200026

Azevedo, R. A. de. (2012). Evaluation of the knowledge of dentists about the approaches adopted across the alveolodentários trauma. Dissertação (Mestrado em Odontologia) - Universidade Federal de Pelotas, Pelotas.

Bastianini, M. E., Restani Oliveira, A., Garrido Mori Panucci, G., dos Santos Santinoni, C., Avansini Marsicano, J., \& Leal do Prado, R. (2021). Traumatismo dentário: qual o conhecimento do cirurgião dentista?. Colloquium Vitae., 12(3), 94-101. https://revistas.unoeste.br/index.php/cv/article/view/3415

Carvalho, Érica dos S., Trindade, R. A., Morais Silva, B. M., Bacelar, L. M., Cangussu, M. C. T., \& Martins, J. D. (2020). Prevalência e complicações das lesões dentárias traumáticas. Revista De Ciências Médicas E Biológicas, 19(3), 394-399. https://doi.org/10.9771/cmbio.v19i3.33002

Cohenca, N., Forrest, J. L., \& Rotstein, I. (2006). Knowledge of oral health professionals of treatment of avulsed teeth. Dental traumatology: official publication of International Association for Dental Traumatology, 22(6), 296-301. https://doi.org/10.1111/j.1600-9657.2005.00448.x

Dantas, V. B., Alves, A. C., \& Scavuzzi, A. I. F. (2019). Prevalência de trauma dental em crianças e adolescentes atendidos no NEPTI da FOUFBA. Revista Da ABENO, 19(2), 71-81. https://doi.org/10.30979/rev.abeno.v19i2.871

Diangelis, A. J., Andreasen, J. O., Ebeleseder, K. A., Kenny, D. J., Trope, M., Sigurdsson, A., Andersson, L., Bourguignon, C., Flores, M. T., Hicks, M. L., Lenzi, A. R., Malmgren, B., Moule, A. J., Pohl, Y., Tsukiboshi, M., \& International Association of Dental Traumatology (2012). International Association of Dental Traumatology guidelines for the management of traumatic dental injuries: 1. Fractures and luxations of permanent teeth. Dental traumatology: official publication of International Association for Dental Traumatology, 28(1), 2-12. https://doi.org/10.1111/j.1600-9657.2011.01103.x

Flores, M. T., Andreasen, J. O., Bakland, L. K., Feiglin, B., Gutmann, J. L., Oikarinen, K., Pitt Ford, T. R., Sigurdsson, A., Trope, M., Vann, W. F., Jr, Andreasen, F. M., \& International Association of Dental Traumatology (2001). Guidelines for the evaluation and management of traumatic dental injuries. Dental traumatology: official publication of International Association for Dental Traumatology, 17(5), 193-198. https://oi.org/10.1034/j.16009657.2001.170501.x

Losso E. M, Tavares M. C. R, Bertoli F. M. P \& Baratto-Filho F. (2011) Traumatismo dentoalveolar na dentição decídua. RSBO, 8(1): 1-20. http://revodonto.bvsalud.org/pdf/rsbo/v8n1/a19v8n1.pdf

Moterle, C., Benemann, M. P., Cavalheiro, S., Tomasi, A. M., Dirschnabel, A. J., Muniz, M., \& Imanishi, S. A. watanabe. (2016). O trauma oclusal na odontologia. Ação Odonto, (1). https://portalperiodicos.unoesc.edu.br/acaodonto/article/view/10440

Pacheco, L. P. (2016). Acompanhamento Radiográfico De Trauma Dental: Um Relato De Caso Clínico. (Monografia). Porto Alegre: Universidade Federal do Rio Grande do Sul, 2016. https://lume.ufrgs.br/handle/10183/150285

Panzarini, S. R., Pedrini, D., Poi, W. R., Sonoda, C. K., Brandini, D. A., \& Monteiro de Castro, J. C. (2008). Dental trauma involving root fracture and periodontal ligament injury: a 10-year retrospective study. Brazilian oral research, 22(3), 229-234. https://doi.org/10.1590/s1806-83242008000300007

Pedrini, D. (2008). Análise do conhecimento dos cirurgiões dentistas sobre plano de tratamento das injúrias do ligamento periodontal após traumatismo dentoalveolar. (Tese - Livre-Docência). Araçatuba: Faculdade de Odontologia da Universidade Estadual Paulista. https://repositorio.unesp.br/handle/11449/106717?show=full

Queiroz, F. de S., Costa, L. E. D., Silva, G. M. da, \& Régis, M. de A. (2019). Perfil e conduta dos cirurgiões-dentistas de Patos-PB frente aos traumatismos dentoalveolares. ARCHIVES OF HEALTH INVESTIGATION, 8(7). https://doi.org/10.21270/archi.v8i7.3803

Sanabe, M. E., Cavalcante, L. B., Coldebella, C. R. \& Abreu-e-Lima, F. C. B. (2009). Urgências em traumatismos dentários: classificação, características e procedimentos. Rev. Paulista de Pediatriatria, São Paulo , 27(4), 447-451. https://doi.org/10.1590/S0103-05822009000400015

Santos, C. A. O., Caldas, A. T. L., Souza, R. L. F., Siewes, M., Carvalho, M. M. P. \& Campos, F. A. T. (2018). Traumatismos dentários: conhecimento dos cirurgiões dentistas lotados na atenção básica de saúde. REFACS, 6(2), 174-180. 10.18554 / refacs.v6i2.2812 
Research, Society and Development, v. 10, n. 16, e394101623819, 2021

(CC BY 4.0) | ISSN 2525-3409 | DOI: http://dx.doi.org/10.33448/rsd-v10i16.23819

Santos, J. F. D., de Medeiros Silva, L. R., Souza, E. R. L. de, Alves, N. M., \& de Almeida Pinto Sarmento, T. C. (2020). Influência do traumatismo dentário na qualidade de vida de crianças de 8 a 10 anos do município de Patos - PB, Brasil. ARCHIVES OF HEALTH INVESTIGATION, 10(2), 241-247. https://doi.org/10.21270/archi.v10i2.4857

Souza, B. L. M. de, Lopes, P. H. de S., Nogueira, E. F. de C. \& Torres, B. C. A. (2013). Manejo de trauma dentoalveolar atípico: relato de caso. Rev. cir. traumatol. buco-maxilo-fac. [online], 13(4), 45-50. http://revodonto.bvsalud.org/scielo.php?script=sci_arttext\&pid=S1808-52102013000400007

Zangirolami-Raimundo, J., Echeimberg, J. O., \& Leone, C. Research methodology topics: Cross-sectional studies.Journal of Human Growth and Development. 2018, 28(3):356-360. http://dx.doi.org/10.7322/jhgd.152198 